\title{
Determination of ascorbic acid by perturbation of the oscillation pattern of the Belousov-Zhabotinskii reaction using a continuous flow system
}

\section{Determinação de ácido ascórbico por perturbação do padrão de oscilação da reação de Belousov-Zhabotinskii utilizando sistema em fluxo contínuo}

Article Info:

Article history: Received 2021-08-26 / Accepted 2021-12-21 / Available online 2021-12-21

doi: $10.18540 /$ jcecvl7iss4pp13622-01-13e

\author{
Patrícia Xavier Baliza \\ ORCID: https://orcid.org/0000-0002-5870-9659 \\ Universidade Federal dos Vales do Jequitinhonha e Mucuri \\ E-mail: patricia.baliza@ufvjm.edu.br \\ Efraim Lázaro Reis \\ ORCID: https://orcid.org/0000-0003-4285-8892 \\ Universidade Federal de Viçosa \\ E-mail: efraimreis@gmail.com \\ César Reis \\ ORCID: https://orcid.org/0000-0002-4783-9634 \\ Universidade Federal de Viçosa \\ E-mail: cesareis2011@gmail.com \\ Ana Paula Lelis Rodrigues \\ ORCID: https://orcid.org/0000-0003-3108-9661 \\ Instituto Federal do Sudeste de Minas Gerais \\ E-mail: ana.rodrigues@ifsudestemg.edu.br \\ Clausius Duque Gonçalves Reis \\ ORCID: https://orcid.org/0000-0002-7422-2066 \\ Universidade Federal de Viçosa \\ E-mail: clausiusreis@gmail.com
}

\section{Resumo}

Propõe-se a determinação potenciométrica de ácido ascórbico (vitamina C) em medicamentos, em um sistema em fluxo, utilizando-se como princípio o efeito desta substância no padrão de oscilação da reação de Belousov-Zhabotinskii. Esta reação envolve a oxidação de um composto orgânico, como o ácido malônico, por íons bromato em meio de ácido sulfúrico, com o íon cério (IV) usado como catalisador. Tanto a frequência como a amplitude das oscilações podem ser usadas como parâmetros analíticos. O ácido ascórbico pode promover a diminuição da diferença de potencial, aumentando a amplitude na reação de Belousov-Zhabotinskii quando esta substância é injetada no meio reacional. $\mathrm{O}$ sistema químico é perturbado com quantidades variáveis de ácido ascórbico, o que resulta em um aumento da amplitude que é linearmente proporcional à concentração de ácido ascórbico. A maioria dos íons comumente encontrados em amostras de medicamentos não interfere na determinação. As condições experimentais foram escolhidas através de um planejamento fatorial $2^{4}$, onde as concentrações dos reagentes bromato de potássio, sulfato de cério (IV), ácido malônico e ácido sulfúrico foram as variáveis estudadas. A metodologia para a determinação de ácido ascórbico resultou em um procedimento relativamente simples, com limite de detecção de 1,63 x $10^{-4} \mathrm{~mol} \mathrm{~L}{ }^{-1}$. Na determinação de ácido ascórbico em amostras de medicamentos obteve-se resultados em concordância com os valores declarados pelos fabricantes. O método desenvolvido 
apresenta-se como uma alternativa para a determinação de ácido ascórbico em medicamentos, pois o procedimento é relativamente simples e de baixo custo.

Palavras-chave: Ácido ascórbico. Reações oscilantes. Sistema em fluxo. Reação de BelousovZhabotinskii.

\section{Abstract}

It is proposed the potentiometric determination of ascorbic acid (vitamin C) in medicines, in a flow system, using as principle the effect of this substance in the oscillation pattern of the BelousovZhabotinskii reaction. This reaction involves the oxidation of an organic compound, such as malonic acid, by bromate ions in sulfuric acid medium, with the cerium (IV) ion used as a catalyst. Both frequency and amplitude of oscillations can be used as analytical parameters. Ascorbic acid can reduce the potential difference, increasing the amplitude of the Belousov-Zhabotinskii reaction when this substance is injected into the reaction medium. The chemical system is disturbed with varying amounts of ascorbic acid, which results in an increase in amplitude that is linearly proportional to the concentration of ascorbic acid. Most ions commonly found in drug samples do not interfere with the determination. The experimental conditions were chosen through a $2^{4}$ factorial design, where the concentrations of the reagents potassium bromate, cerium (IV) sulphate, malonic acid and sulfuric acid were the studied variables. The methodology for the determination of ascorbic acid resulted in a relatively simple procedure, with a detection limit of $1.63 \times 10^{-4} \mathrm{~mol} \mathrm{~L}^{-1}$. In the determination of ascorbic acid in drug samples, results were obtained in accordance with the values declared by the manufacturers. The developed method presents itself as an alternative for the determination of ascorbic acid in medications, as the procedure is relatively simple and inexpensive. Keywords: Ascorbic acid. Oscillating reactions. Flow system. Belousov-Zhabotinskii reaction.

\section{Introdução}

O ácido ascórbico, mais conhecido como vitamina C, é uma substância solúvel em água que participa de numerosos processos biológicos. Ocorre em uma variedade de alimentos naturais, como frutas, verduras e hortaliças. Este ácido é adicionado como antioxidante a vários produtos farmacêuticos ou como estabilizante para complexos da vitamina B. O grande uso de produtos naturais e farmacêuticos contendo ácido ascórbico levou ao desenvolvimento de procedimentos analíticos para a sua determinação.

A grande maioria dos métodos propostos para determinação de ácido ascórbico, espectrofotométricos, cromatográficos ou titrimétricos, é aplicada a amostras que incluem medicamentos, sucos, bebidas não-alcóolicas, soro sanguíneo e alimentos (ALDRIGUE, 1998; AYDOGMUS e CETIN, 2002; EMADI-KONJIN et al., 2005; OLIVEIRA et al., 2010).

Em geral, procedimentos com detecção espectrofotométrica apresentam interferências mais sérias, principalmente quando analisadas amostras coloridas contendo compostos que absorvem a radiação na mesma região espectral que o produto analisado e desta forma exigem pre-tratamentos tediosos e demorados (AYDOGMUS e CETIN, 2002).

Uma alternativa é o método potenciométrico utilizando o sistema oscilante de BelousovZhabotinskii (BZ) que envolve três reações globais, as equações 1 e 2 representam os principais processos, que alternadamente controlam a reação de BZ, resultando em oscilações na concentração da espécie intermediaria (FARIA, 1995; OJANI, 2003).

$$
\begin{gathered}
\mathrm{BrO}_{3}^{-}+2 \mathrm{Br}^{-}+3 \mathrm{CH}_{2}(\mathrm{COOH})_{2}+3 \mathrm{H}^{+} \rightarrow 3 \mathrm{BrCH}(\mathrm{COOH})_{2}+3 \mathrm{H}_{2} \mathrm{O} \\
\mathrm{BrO}_{3}{ }^{-}+4 \mathrm{Ce}^{3+}+5 \mathrm{H}^{+} \rightarrow \mathrm{HBrO}+4 \mathrm{Ce}^{4+}+2 \mathrm{H}_{2} \mathrm{O} \\
\mathrm{BrCH}(\mathrm{COOH})_{2}+4 \mathrm{Ce}^{4+}+\mathrm{HBrO}+\mathrm{H}_{2} \mathrm{O} \rightarrow 2 \mathrm{Br}^{-}+3 \mathrm{CO}_{2}+4 \mathrm{Ce}^{3+}+6 \mathrm{H}^{+}
\end{gathered}
$$

O processo representado pela equação 3 é o vínculo entre os dois processos anteriores. No processo representado pela equação 1 , os íons bromato, brometo e o ácido malônico reagem para 
formar o ácido bromomalônico. Quando a concentração de brometo diminui, ficando abaixo do ponto crítico, o controle da reação muda para o processo representado pela equação 2 , no qual o $\mathrm{Ce}(\mathrm{III})$ é oxidado por bromato; na sequência o $\mathrm{Ce}(\mathrm{IV})$ é reduzido a $\mathrm{Ce}(\mathrm{III})$ pelo ácido bromomalônico, como na equação 3. Quando uma quantidade suficiente de brometo é formada no processo representado pela equação 3, o controle da reação é devolvido para o processo representado na equação 1. A reação continua até a concentração de um dos reagentes diminuir, ficando abaixo do nível necessário para sustentar o ciclo.

No processo global observa-se um período de indução onde ocorre uma lenta oxidação do ácido malônico através do íon bromato, catalisada por cério. Quando é atingida uma concentração crítica do bromato, ele reage com o cério (IV) formando o íon brometo, parando a oxidação e levando a reação de volta ao processo inicial, a oscilação então se inicia (FARIA, 1995).

O método mais utilizado para experimentação com reações oscilantes faz uso do reator de fluxo continuo com agitação. Neste método as soluções e reagentes são continuamente alimentadas ao reator por meio de uma bomba peristáltica. O volume da solução é mantido constante fazendose com que o excesso transborde por uma saída ou ainda retirado pelo uso de uma conexão a uma bomba peristáltica. A principal vantagem deste método é o estudo do formato das oscilações por longo tempo e também a facilidade de verificar modificações nas oscilações provocadas pelas condições experimentais (MAIA et al., 2019; RAOOF, 2004)).

Propõe-se assim um método potenciométrico para a determinação de ácido ascórbico (vitamina C), em um sistema em fluxo contínuo (PAIM, 2002; YEBRA-BIURRUN, 2000), baseado no efeito desta substância na reação de Belousov-Zhabotinskii. O ácido ascórbico é bastante conhecido pelo seu poder anti-oxidante (AL-GHANNAM e AL-OLYAN, 2005; BUENO, 2019; YEBRA-BIURRUN, 2000). Em determinadas faixas de concentração, este ácido pode reagir com cério (IV) para formar um complexo, conforme a equação 4.

Assim pela complexação, o ácido ascórbico inibe a redução de $\mathrm{Ce}(\mathrm{IV})$ a $\mathrm{Ce}(\mathrm{III})$, o que resulta em um decréscimo na concentração de brometo, de acordo com a equação 3 . O aumento da amplitude resulta do decréscimo da razão $\mathrm{Ce}(\mathrm{IV}) / \mathrm{Ce}(\mathrm{III})$, enquanto o período decresce devido a uma baixa taxa de geração de íons brometo.

\section{Materiais e Métodos}

\subsection{Instrumentação}

Todas as determinações foram realizadas utilizando-se um potenciômetro Tecnopar, modelo mPA210 e um eletrodo de referência de prata-cloreto de prata com dupla junção, da marca Orion modelo 90020, tendo o compartimento externo preenchido com uma solução de $\mathrm{KNO}_{3}$ a $(10 \% \mathrm{~m} / \mathrm{v})$ e um eletrodo indicador de platina da marca Beckman. O potenciômetro foi interfaceado serialmente via RS232C e o gerenciamento e aquisição de dados feita por um programa em QuickBasic v.4.5.<smiles>CC(C)[C@H](C)CO</smiles> 


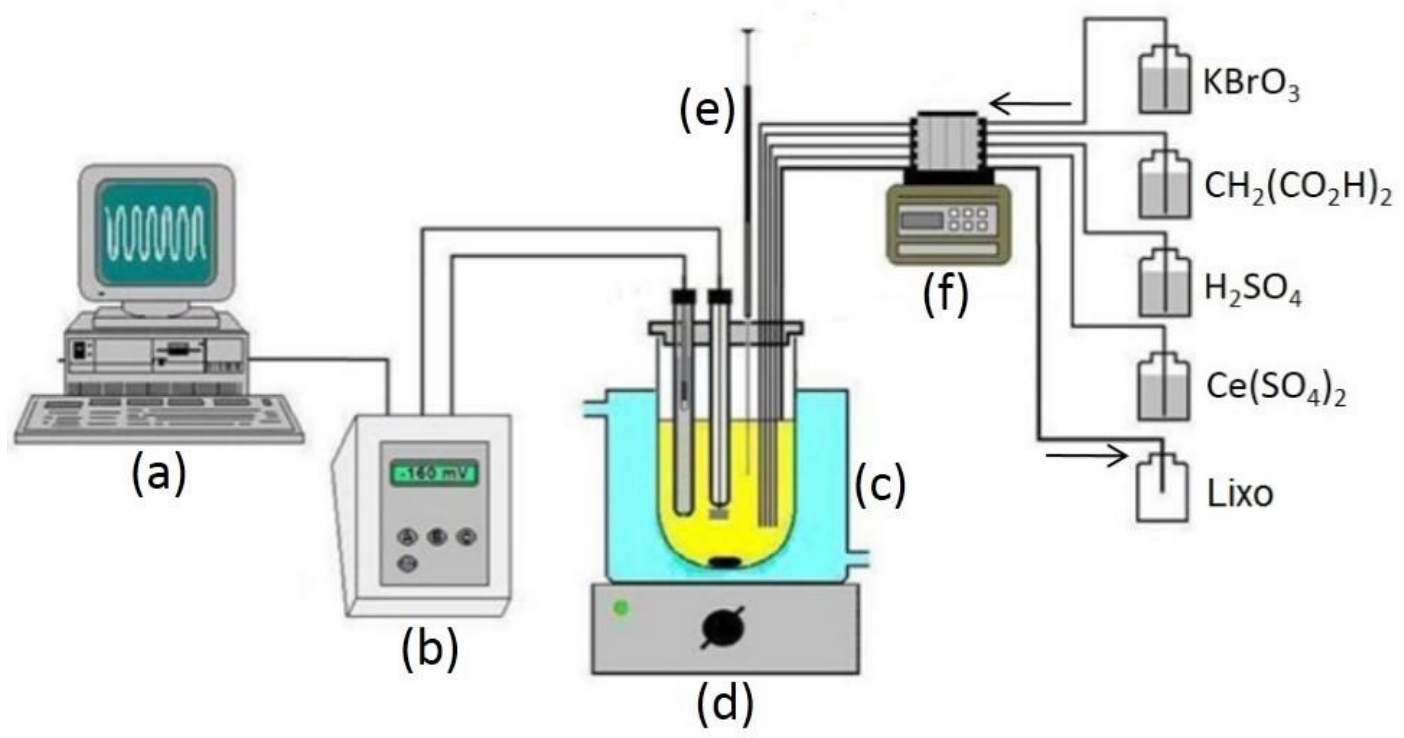

Figura 1 - Sistema completo do instrumental. Microcomputador (a); Potenciômetro (b); Cela termostatizada com os eletrodos de platina, de referência de $\mathrm{Ag} / \mathrm{AgCl}$ (c); Agitador magnético (d); Micropipeta (e); Bomba peristáltica (f). (Fonte: Os autores, 2020)

Um banho termostatizado da marca Frigomix, modelo B, foi utilizado para controle da temperatura reacional. $\mathrm{O}$ transporte dos reagentes, por capilares de polietileno, foi realizado com o auxílio de uma bomba peristáltica Minipuls 3, com 8 canais e tubos de bombeamento de tygon ${ }^{\circledR}$. Um agitador magnético da marca Corning, modelo PC-320 foi empregado para a homogeneização do meio reacional, de acordo com a figura 1.

\subsection{Soluções e reagentes}

Todos os reagentes utilizados na realização deste trabalho foram de grau analítico, não tendo sido submetidos a qualquer tratamento adicional. As soluções foram preparadas com água deionizada. Toda a vidraria foi devidamente limpa com detergente e água deionizada, exceto as vidrarias utilizadas no preparo das soluções para o estudo de interferentes, que foram deixadas em ácido nítrico a $5 \%(\mathrm{~m} / \mathrm{v})$ durante 24 horas e posteriormente lavadas com água deionizada.

As soluções estoques de sulfato de cério (IV) $0,10 \mathrm{~mol} \mathrm{~L}^{-1}$, bromato de potássio $(0,40 \mathrm{~mol}$ $\left.\mathrm{L}^{-1}\right)$, ácido malônico $\left(0,80 \mathrm{~mol} \mathrm{~L}^{-1}\right)$, ácido sulfúrico $\left(1,50 \mathrm{~mol} \mathrm{~L}^{-1}\right)$ e ácido ascórbico $\left(0,10 \mathrm{~mol} \mathrm{~L}^{-1}\right)$ foram preparadas em água deionizada. Para o estudo de interferentes, foram preparadas soluções de $\mathrm{NaNO}_{3}, \mathrm{NaNO}_{2}, \mathrm{CuSO}_{4}, \mathrm{MnSO}_{4}, \mathrm{Al}\left(\mathrm{NO}_{3}\right)_{3}, \mathrm{NaCl}$ e $\mathrm{NH}_{4} \mathrm{SCN}$ também em água deionizada em concentrações variadas.

\subsection{Procedimento geral}

O sistema mostrado na figura 1 foi empregado para o monitoramento do sinal da reação oscilante. As soluções de sulfato de cério (IV), bromato de potássio, ácido malônico e ácido sulfúrico foram adicionadas continuamente na cela, em uma vazão constante de $0,50 \mathrm{~mL} \mathrm{~min}{ }^{-1}$ e a linha denominada lixo, para retirada da solução, foi mantida em uma vazão de $2,00 \mathrm{~mL} \mathrm{~min}{ }^{-1}$ garantindo volume constante e consequentemente concentrações constantes na cela.

$\mathrm{Na}$ determinação de ácido ascórbico foi injetado um volume de $100 \mu \mathrm{L}$ da amostra na cela, com auxílio de uma micropipeta mostrada na figura 1. 


\subsection{Planejamento fatorial}

A escolha das melhores concentrações dos reagentes, para que o sistema oscilasse de forma regular, foi realizada através de um planejamento fatorial completo $2^{4}$. Os reagentes bromato de potássio, sulfato de cério (IV), ácido malônico e ácido sulfúrico foram as variáveis estudadas no planejamento. Os valores mínimo e máximo destas variáveis são mostrados na tabela 1 . A tabela 2 mostra o planejamento simples $2^{4}$ realizado. A influência da temperatura no sistema foi estudada posteriormente após a escolha das melhores condições dos reagentes.

Tabela 1 - Valores mínimos e máximos das variáveis estudadas no planejamento $2^{4}$.

\begin{tabular}{ccc}
\hline \multirow{2}{*}{ Variável } & \multicolumn{2}{c}{ Concentração $\left(\mathrm{mol} \mathrm{L}^{-1}\right)$} \\
\cline { 2 - 3 } & Valor mínimo (-) & Valor máximo (+) \\
\hline $\mathrm{KBrO}_{3}$ & 0,2 & 0,4 \\
\hline $\mathrm{Ce}\left(\mathrm{SO}_{4}\right)_{2}$ & 0,004 & 0,01 \\
\hline $\mathrm{CH}_{2}(\mathrm{COOH})_{2}$ & 0,3 & 0,6 \\
\hline $\mathrm{H}_{2} \mathrm{SO}_{4}$ & 0,5 & 1,0 \\
\hline
\end{tabular}

Tabela 2 - Planejamento fatorial $2^{4}$ para a otimização das concentrações dos reagentes.

\begin{tabular}{ccccccccc}
\hline \multirow{2}{*}{ Ensaio } & $\mathrm{X}_{1}$ & $\mathrm{X}_{2}$ & $\mathrm{X}_{3}$ & $\mathrm{X}_{4}$ & $\mathrm{KBrO}_{3}$ & $\mathrm{Ce}\left(\mathrm{SO}_{4}\right)_{2}$ & $\mathrm{CH}_{2}(\mathrm{COOH})_{2}$ & $\mathrm{H}_{2} \mathrm{SO}_{4}$ \\
\cline { 2 - 10 } & \multicolumn{6}{c}{ Variáveis codificadas } & \multicolumn{7}{c}{$\mathrm{mol} \mathrm{L}^{-1}$} \\
\hline 1 & + & + & + & + & 0,4 & 0,010 & 0,6 & 1,0 \\
\hline 2 & + & + & + & - & 0,4 & 0,010 & 0,6 & 0,5 \\
\hline 3 & + & + & - & + & 0,4 & 0,010 & 0,3 & 1,0 \\
\hline 4 & + & + & - & - & 0,4 & 0,010 & 0,3 & 0,5 \\
\hline 5 & + & - & + & + & 0,4 & 0,004 & 0,6 & 1,0 \\
\hline 6 & + & - & + & - & 0,4 & 0,004 & 0,6 & 0,5 \\
\hline 7 & + & - & - & + & 0,4 & 0,004 & 0,3 & 1,0 \\
\hline 8 & + & - & - & - & 0,4 & 0,004 & 0,3 & 0,5 \\
\hline 9 & - & + & + & + & 0,2 & 0,010 & 0,6 & 1,0 \\
\hline 10 & - & + & + & - & 0,2 & 0,010 & 0,6 & 0,5 \\
\hline 11 & - & + & - & + & 0,2 & 0,010 & 0,3 & 1,0 \\
\hline 12 & - & + & - & - & 0,2 & 0,010 & 0,3 & 0,5 \\
\hline 13 & - & - & + & + & 0,2 & 0,004 & 0,6 & 1,0 \\
\hline 14 & - & - & + & - & 0,2 & 0,004 & 0,6 & 0,5 \\
\hline 15 & - & - & - & + & 0,2 & 0,004 & 0,3 & 1,0 \\
\hline 16 & - & - & - & - & 0,2 & 0,004 & 0,3 & 0,5 \\
\hline & & & & & & & & \\
\hline
\end{tabular}




\subsection{Preparo das amostras}

Foram analisadas duas amostras comerciais de medicamentos. As amostras consistiam de comprimidos e de material em pó, ambos solúveis em água. Para a determinação foram pesadas 4,000 g de amostras de comprimido e 1,000 g de amostras em pó, dissolvidas em água deionizada e transferidas para balões volumétricos de $100 \mathrm{~mL}$ completando-se o volume com água deionizada. Para a amostra do comprimido, foi feita uma diluição de $25 \mathrm{~mL}$ da solução inicial para um volume de $100 \mathrm{~mL}$, devido à maior concentração de ácido ascórbico.

\section{Resultados e discussão}

\subsection{Cálculo da amplitude e da frequência}

Como exemplo, as oscilações regulares, resultado do experimento 5 do planejamento fatorial, são mostradas na figura 2. Os pontos A e B correspondem aos tempos entre 250 e $850 \mathrm{~s}$, sendo o cálculo da amplitude e da frequência realizado sempre entre esses dois valores. A este espaço de tempo convencionou-se chamar de janela analítica. Os pontos $\mathrm{M}$ e $\mathrm{N}$, também mostrados na figura 2, são os pontos inicial e final, respectivamente, para a tomada de valores da amplitude para cada ciclo, calculada da seguinte forma (Equação 5):

$$
\Delta \mathrm{A}=|\mathrm{M}-\mathrm{N}|
$$

onde $\Delta \mathrm{A}$ é a amplitude em cada ciclo. Para a obtenção da amplitude média das oscilações, foram utilizados todos os ciclos oscilatórios dentro da janela analítica.

Os pontos $\mathrm{N}_{1}, \mathrm{~N}_{2}, \ldots, \mathrm{N}_{\mathrm{n}}$ mostrados na figura 2 são os máximos de cada ciclo de oscilação. $\mathrm{O}$ intervalo $\mathrm{T}$ é o período de oscilação entre dois máximos. O período para cada ciclo foi calculado utilizando-se a Equação 6. A frequência calculada é o inverso do período. A frequência média das oscilações foi obtida com todos os ciclos oscilatórios dentro da janela analítica.

$$
\mathrm{T}=\mathrm{N}_{\mathrm{n}}-\mathrm{N}_{\mathrm{n}-1}
$$

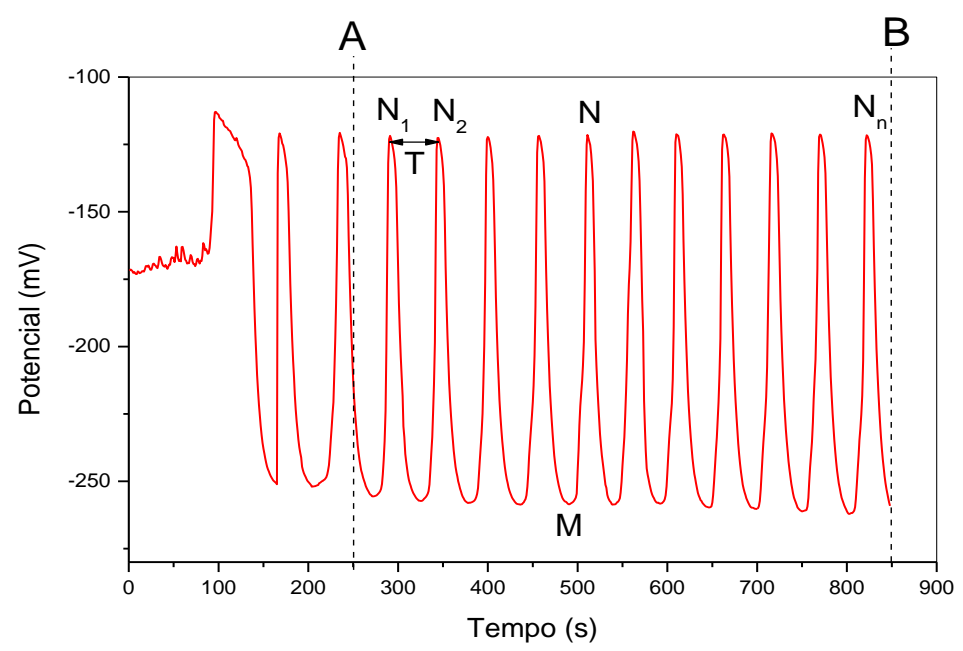

Figura 2 - Padrão de oscilação da reação de Belouzov-Zhabotinskii.

A figura 3 mostra os gráficos de oscilação do sistema BZ correspondentes aos experimentos de 1 a 16 do planejamento fatorial. Nos experimentos de 1 a 7 ocorreram oscilações regulares e para estes experimentos foram calculadas a amplitude e a frequência. Os resultados destes cálculos são mostrados na tabela 3 . Nos experimentos $1 \mathrm{e} 3$, os valores dos desvios padrão da amplitude são mais elevados, devido à instabilidade facilmente perceptível em alguns 
pontos da janela analítica. Optou-se pelas condições do experimento 5, por apresentar um alto valor de amplitude e valores baixos dos desvios padrão para a amplitude e para a frequência.
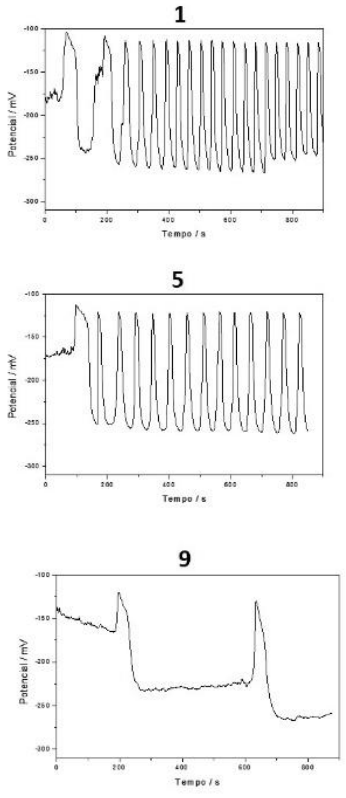

13

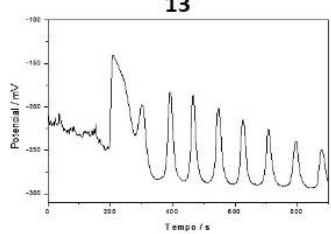

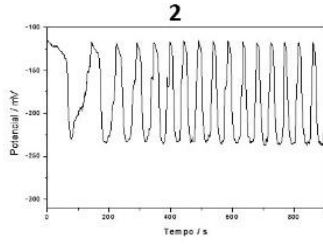

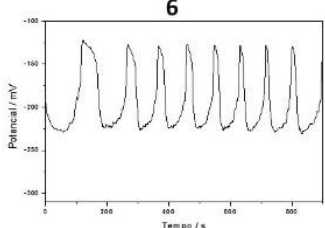

10

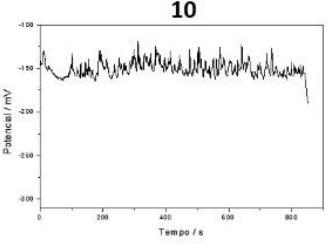

14

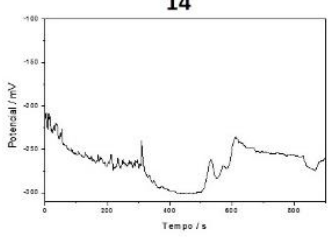

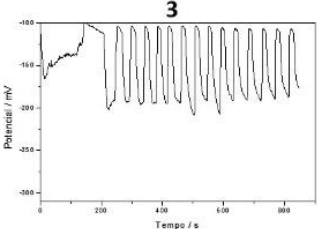
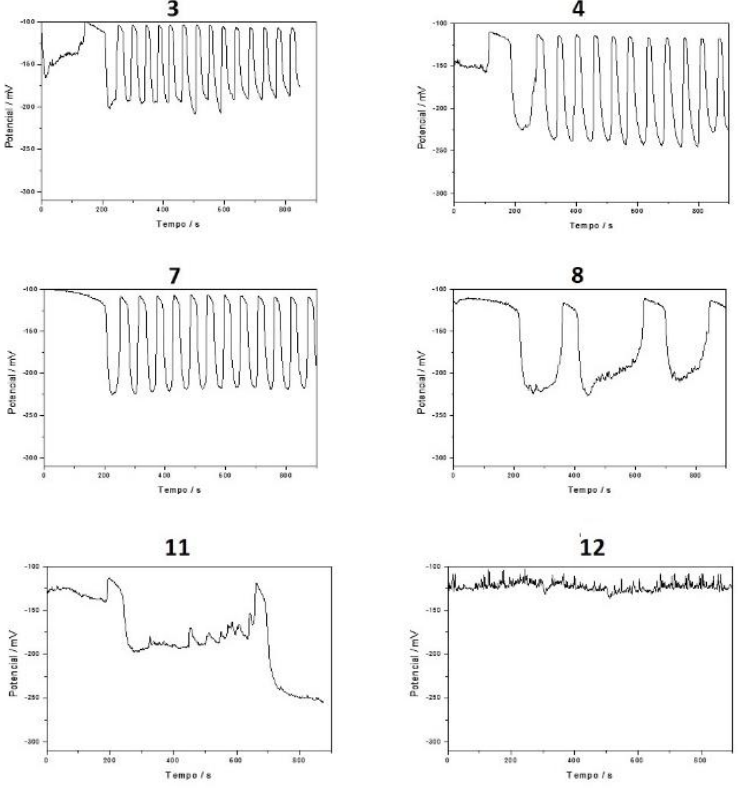

15

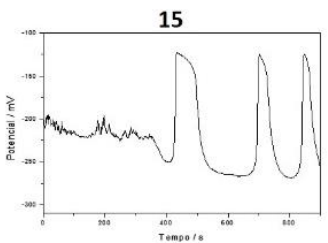

16

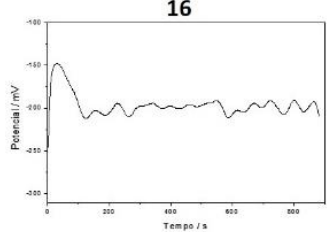

Figura 3 - Gráficos de oscilação do sistema BZ correspondentes aos experimentos 1 a 16 do planejamento fatorial.

Tabela 3 - Amplitude média, frequência média e desvio padrão na janela analítica, para as misturas com oscilações regulares.

\begin{tabular}{ccc|cc}
\hline Experimento & $\begin{array}{c}\text { Amplitude } \\
(\mathrm{mV})\end{array}$ & $\begin{array}{c}\text { Desvio } \\
\text { Padrão }\end{array}$ & $\begin{array}{c}\text { Frequência } \\
\left(\mathrm{s}^{-1}\right)\end{array}$ & $\begin{array}{c}\text { Desvio } \\
\text { Padrão }\end{array}$ \\
\hline 1 & 145,412 & 7,264 & 37,000 & 4,163 \\
\hline 2 & 118,018 & 1,223 & 47,636 & 3,471 \\
\hline 3 & 89,407 & 7,048 & 43,916 & 3,449 \\
\hline 4 & 125,677 & 2,224 & 59,555 & 4,156 \\
\hline 5 & 136,891 & 2,507 & 53,100 & 2,514 \\
\hline 6 & 98,0830 & 1,371 & 89,166 & 6,790 \\
\hline 7 & 110,920 & 2,678 & 56,400 & 2,270 \\
\hline
\end{tabular}

\subsection{Influência da concentração de bromato de potássio}

Neste estudo, foi variada a concentração de bromato de potássio, empregando-se os valores 0,$10 ; 0,20 ; 0,25 ; 0,30 ; 0,35$ e $0,40 \mathrm{~mol} \mathrm{~L}^{-1}$. As concentrações de ácido sulfúrico, ácido malônico e 
sulfato de cério (IV) foram fixadas em 1,0;0,60 e 0,004 mol L-1, respectivamente. A figura 5(a) mostra a variação da amplitude de oscilação com a concentração de bromato de potássio. De acordo com o gráfico, a amplitude é máxima quando utilizados valores de concentração maiores ou iguais a $0,35 \mathrm{~mol} \mathrm{~L}^{-1}$.

Não foram estudadas concentrações de bromato de potássio maiores que $0,40 \mathrm{~mol} \mathrm{~L}^{-1}$ devido à limitação imposta pela solubilidade deste sal. Desta forma, foi utilizada uma concentração de 0,40 mol L $\mathrm{L}^{-1}$ nos experimentos posteriores, pois o valor de $0,35 \mathrm{~mol} \mathrm{~L}^{-1}$ ficaria em uma região onde qualquer decréscimo na concentração resultaria em diminuição da amplitude.

\subsection{Influência da concentração de sulfato de cério (IV)}

Fixado o valor da concentração de bromato de potássio em $0,40 \mathrm{~mol} \mathrm{~L}^{-1}$, foi estudada a influência da concentração de sulfato de cério (IV). As concentrações das soluções de ácido sulfúrico $\left(1,00 \mathrm{~mol} \mathrm{~L}^{-1}\right)$ e ácido malônico $\left(0,60 \mathrm{~mol} \mathrm{~L}^{-1}\right)$ foram novamente fixadas. A concentração de sulfato de cério (IV) foi variada nos valores $0,002,0,004 ; 0,006 ; 0,008 ; 0,01$ e $0,012 \mathrm{~mol} \mathrm{~L}^{-1}$. A influência da concentração de sulfato de cério (IV) no sistema BZ é mostrada na figura 5(b).

Pode ser observado que melhores resultados são obtidos quando utilizados valores de concentração na faixa de 0,006 a $0,009 \mathrm{~mol} \mathrm{~L}^{-1}$. Em todos os experimentos posteriores foi utilizada, então, solução de sulfato de cério (IV) na concentração $0,008 \mathrm{~mol} \mathrm{~L}^{-1}$.

\subsection{Influência da concentração de ácido malônico}

Foram preparadas soluções de ácido malônico nas concentrações de: 0,30, 0,40, 0,50, 0,60; 0,70 e $0,80 \mathrm{~mol} \mathrm{~L}^{-1}$. Como nos experimentos anteriores foram mantidas as concentrações de bromato de potássio em $0,4 \mathrm{~mol} \mathrm{~L}^{-1}$, de sulfato de cério (IV) em $0,008 \mathrm{~mol} \mathrm{~L}^{-1}$ e de ácido sulfúrico em 1,00 $\mathrm{mol} \mathrm{L}{ }^{-1}$.

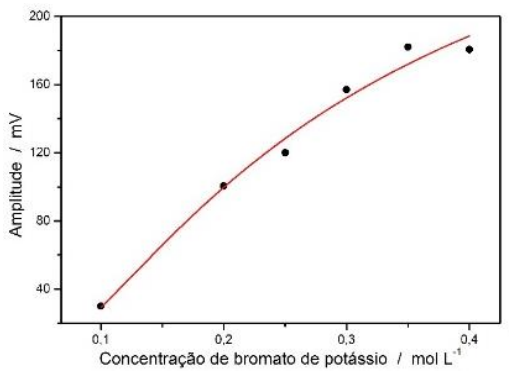

(a)

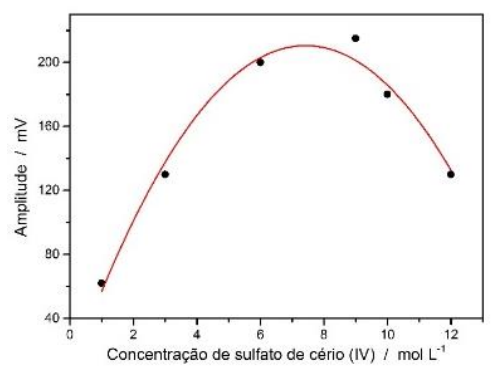

(b)

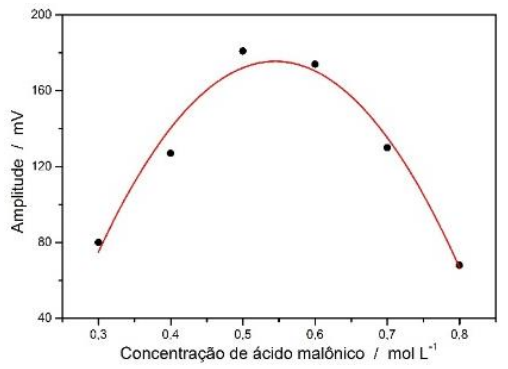

(c)

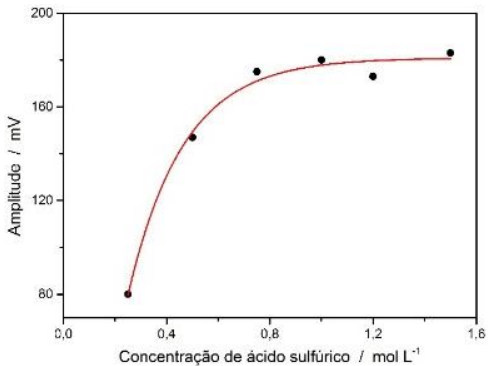

(d)

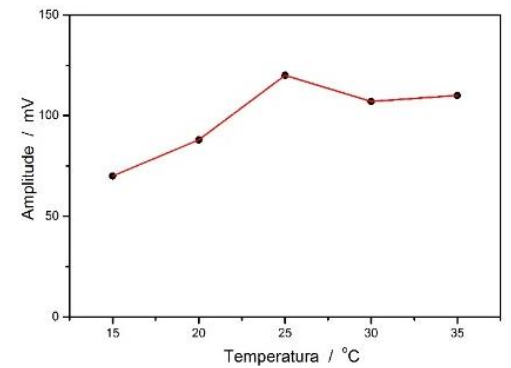

(e)

Figura 5 - Influência das concentrações dos reagentes bromato de potássio (a), sulfato de cério (b), ácido malônico (c), ácido sulfúrico (d) e da temperatura (e), na amplitude das oscilações e da temperatura (f) na frequência das oscilações. 
Os resultados são apresentados na figura 5(c). Observa-se que a amplitude de oscilação é máxima quando utilizados valores de concentração de ácido malônico na faixa de 0,50 a $\quad 0,60$ mol L ${ }^{-1}$. Desta forma, nos estudos subsequentes, foi empregada solução de ácido malônico de concentração $0,55 \mathrm{~mol} \mathrm{~L}^{-1}$.

\subsection{Influência da concentração do ácido sulfúrico}

Para finalizar o estudo univariado da influência das concentrações dos reagentes utilizados no sistema BZ, foi variada a concentração do ácido sulfúrico, preparando-se soluções de 0,$25 ; 0,50$; 0,$75 ; 1,00 ; 1,20$ e $1,50 \mathrm{~mol} \mathrm{~L}^{-1}$. Mantidas, dos experimentos anteriores, as concentrações de bromato de potássio em $0,4 \mathrm{~mol} \mathrm{~L}^{-1}$, de sulfato de cério (IV) em $0,008 \mathrm{~mol} \mathrm{~L}^{-1}$ e de ácido malônico em 0,55 $\mathrm{mol} \mathrm{L}-1$.

Os resultados demonstraram que, para concentrações maiores ou iguais a $0,90 \mathrm{~mol} \mathrm{~L}^{-1}$, a amplitude de oscilação é máxima, como pode ser observado na figura 5(d). No entanto, quando empregadas soluções do ácido na concentração de $1,20 \mathrm{~mol} \mathrm{~L}^{-1}$ ou mais concentradas, formaram-se bolhas de gás nas paredes da cela e na superfície dos eletrodos. Esse fenômeno causou uma alteração na base dos picos, aumentando, assim, a amplitude de oscilação. Desta forma, foi utilizada solução de ácido sulfúrico $1,00 \mathrm{~mol} \mathrm{~L}^{-1} \mathrm{em}$ todos os experimentos posteriores.

\subsection{Influência da Temperatura no Sistema BZ}

Estabelecidas às concentrações de todos os reagentes no sistema BZ, procedeu-se ao estudo da influência da temperatura. Para este estudo, variou-se a temperatura do meio reacional, através de banho termostatizado. A temperatura foi variada nos valores $15 ; 20 ; 25 ; 30$ e $35{ }^{\circ} \mathrm{C}$, sendo monitorada com um termômetro eletrônico, ligado ao potenciômetro, inserido na cela externa.

Os resultados destes experimentos são mostrados na figura 5(e). Observa-se que, utilizandose uma temperatura de $25{ }^{\circ} \mathrm{C}$, obtém-se um valor de amplitude ligeiramente maior que pode ter ocorrido devido a uma oscilação de uma variável responsável pelo aumento na amplitude na temperatura de $25^{\circ} \mathrm{C}$ e não um máximo nesta temperatura. No entanto, quando foram empregados os valores de 30 e $35^{\circ} \mathrm{C}$, foi observada a formação de bolhas no meio reacional, resultando em picos irregulares. Desta forma, foi estabelecido o valor de temperatura de $25{ }^{\circ} \mathrm{C}$, para os próximos experimentos, devido a maior facilidade no controle da mesma

Após o estudo da influência das variáveis da reação de Belousov-Zhabotinskii no sistema em fluxo, os valores ótimos são listados na Tabela 4.

Tabela 4 - Variáveis otimizadas para a determinação de ácido ascórbico.

\begin{tabular}{cc}
\hline $\mathrm{KBrO}_{3}\left(\mathrm{~mol} \mathrm{~L}^{-1}\right)$ & 0,40 \\
\hline $\mathrm{Ce}\left(\mathrm{SO}_{4}\right)_{2}\left(\mathrm{~mol} \mathrm{~L}^{-1}\right)$ & 0,008 \\
\hline $\mathrm{CH}_{2}(\mathrm{COOH})_{2}\left(\mathrm{~mol} \mathrm{~L}^{-1}\right)$ & 0,55 \\
\hline $\mathrm{H}_{2} \mathrm{SO}_{4}\left(\mathrm{~mol} \mathrm{~L}^{-1}\right)$ & 1,00 \\
\hline Temperatura $\left({ }^{\circ} \mathrm{C}\right)$ & 25,0 \\
\hline
\end{tabular}

\subsection{Parâmetros analíticos}

O ácido ascórbico pode aumentar significativamente a amplitude de oscilação da reação de BZ, quando injetado no sistema. Esse efeito é proporcional à quantidade de ácido ascórbico presente no meio reacional. Assim, é viável a determinação da vitamina $\mathrm{C}$, através da relação da sua concentração com a amplitude do sistema BZ. Para tanto foram injetadas soluções de ácido 
ascórbico nas concentrações de 0,002, 0,004, 0,006, 0,008 e 0,010 mol L-1. A perturbação promovida pelo ácido ascórbico na resposta analítica pode ser visualizada observando-se o gráfico da figura 6.

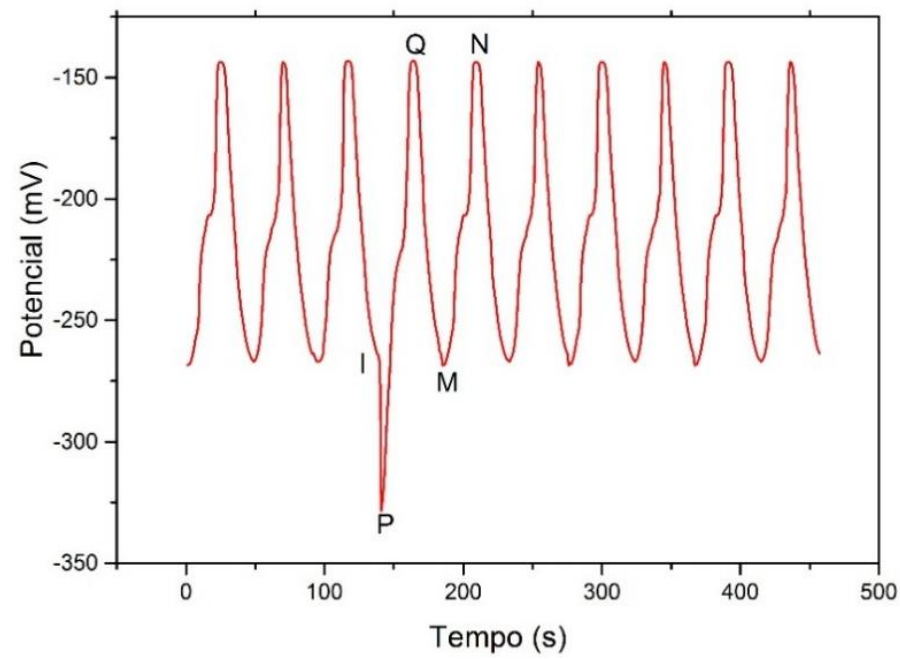

Figura 6 - Gráfico de oscilação do sistema BZ com injeção de ácido ascórbico.

Quando é injetado ácido ascórbico (ponto I), a amplitude do ciclo de injeção aumenta (ponto $\mathrm{P})$, os pontos $\mathrm{P}, \mathrm{Q}, \mathrm{M}$ e $\mathrm{N}$ são medidos em $\mathrm{mV}$, assim a variação da amplitude $(\Delta \mathrm{A})$ pode ser calculada por:

$$
\Delta \mathrm{A}=(\mathrm{P}-\mathrm{Q})-(\mathrm{M}-\mathrm{N}) \quad \text { Equação } 7
$$

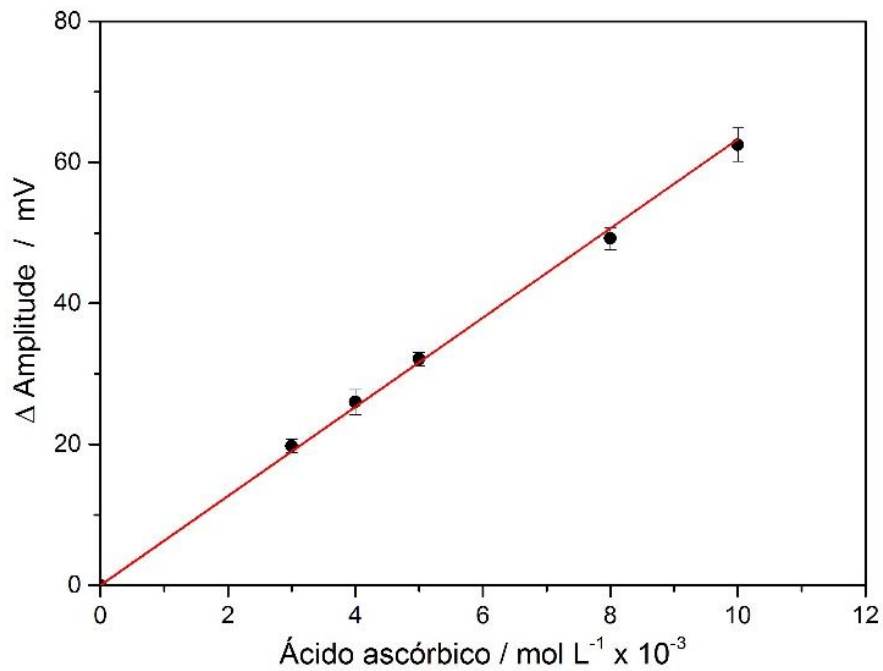

Figura 7 - Curva analítica da determinação de ácido ascórbico utilizando o sistema BZ.

Relacionando-se os valores de $\Delta \mathrm{A}$ com as correspondentes concentrações de ácido ascórbico, obteve-se o gráfico mostrado na figura 7. As características da curva analítica para determinação de ácido ascórbico são mostradas na tabela 5. 
Tabela 5 - Características analíticas da determinação de ácido ascórbico utilizando o sistema BZ. $\Delta \mathrm{A}$, variação na amplitude $(\mathrm{mV}) ; \mathrm{C}$, concentração de ácido ascórbico, $\mathrm{mol} \mathrm{L}^{-1}$.

\begin{tabular}{cc}
\hline Equação da curva & $\Delta \mathrm{A}=0,7635+6165,625 \cdot \mathrm{C}$ \\
\hline Faixa linear $\left(\mathrm{mol} \mathrm{L}^{-1}\right)$ & $1,63 \cdot 10^{-4}-1,00 \cdot 10^{-2}$ \\
\hline Coeficiente de correlação, $\mathrm{r}^{2}$ & 0,9996 \\
\hline Limite de detecção $\left(\mathrm{mol} \mathrm{L}^{-1}\right)$ & $1,63 \cdot 10^{-4}$ \\
\hline
\end{tabular}

\subsection{Estudo de interferentes}

Os sistemas oscilantes podem ser alterados pela presença de espécies interferentes no meio reacional. Desta forma, investigou-se o efeito de algumas dessas substâncias no sistema BZ. Para isso, foram preparadas soluções contendo ácido ascórbico na concentração de $0,005 \mathrm{~mol} \mathrm{~L}^{-1} \mathrm{e}$ concentrações variadas dos possíveis íons interferentes.

Tabela 6 - Interferentes na determinação de ácido ascórbico utilizando o sistema BZ.

\begin{tabular}{|c|c|c|c|}
\hline Substância. & Razão* & Amplitude (mV) & Erro $(\%)$ \\
\hline -----** & & 32,05 & ------- \\
\hline $\mathrm{NaNO}_{3}$ & 300 & 33,40 & 4,2 \\
\hline $\mathrm{NaNO}_{2}$ & 300 & 32,07 & 2,1 \\
\hline $\mathrm{NH}_{4} \mathrm{SCN}$ & 300 & 30,93 & $-3,5$ \\
\hline $\mathrm{MnSO}_{4}$ & 100 & 31,02 & $-3,2$ \\
\hline $\mathrm{NaCl}$ & 100 & 31,66 & $-1,2$ \\
\hline $\mathrm{Al}\left(\mathrm{NO}_{3}\right)_{3}$ & 50 & 32,82 & 2,4 \\
\hline $\mathrm{CuSO}_{4}$ & 50 & 33,49 & 4,5 \\
\hline
\end{tabular}

* Razão máxima tolerada (Substância/Ácido ascórbico)

** Somente ácido ascórbico $0,005 \mathrm{~mol} \mathrm{~L}^{-1}$.

O efeito de cada espécie foi considerado interferência quando ocorreu uma variação acima de $5 \%$ em relação ao sinal analítico do ácido ascórbico sem a presença do interferente. As quantidades (relação interferente/ácido ascórbico) máximas de cada substância, que não causaram interferência são apresentadas na tabela 6 , que mostra que a seletividade do procedimento é aceitável.

\subsection{Aplicação em amostras reais}

Duas amostras comerciais foram preparadas em quadruplicada e injetadas na cela, conforme procedimento descrito. Os resultados são mostrados nas tabelas 7. Estes resultados demostram a aplicabilidade do procedimento à determinação de ácido ascórbico em amostras reais de medicamentos. 
Tabela 7 - Comparação entre os valores descritos pelos fabricantes e os resultados da determinação de ácido ascórbico em amostras de medicamentos utilizando o sistema BZ.

\begin{tabular}{cccc}
\hline Amostra & Apresentação & Declarado & Este método \\
\hline $\begin{array}{c}\text { Vitargyl C } \\
(\mathrm{mg} / 4,0 \mathrm{~g})\end{array}$ & Comprimido & 1000 & $962,00 \pm 22$ \\
\hline $\begin{array}{c}\text { Cebion } \\
(\mathrm{mg} / 1,0 \mathrm{~g})\end{array}$ & Pó & 100 & $98,00 \pm 6$ \\
\hline
\end{tabular}

\section{Conclusões}

A perturbação causada pelo ácido ascórbico ao sistema Belousov-Zhabotinskii resulta em um decréscimo na amplitude da oscilação proporcional à sua concentração. Essa relação foi utilizada com sucesso na determinação do ácido ascórbico em medicamentos.

A determinação de ácido ascórbico (vitamina C) nas amostras analisadas mostrou que os resultados encontrados estão em concordância com os valores declarados pelos fabricantes. O método desenvolvido apresenta-se como uma boa alternativa à determinação de ácido ascórbico em produtos farmacêuticos, pois o procedimento é simples e de baixo custo e os possíveis interferentes não estão presentes, nos tipos de amostras analisadas.

Assim, é bastante pertinente e promissor o estudo da aplicação de sistemas oscilantes em determinações analíticas, visando a dosagem de substâncias orgânicas e inorgânicas.

\section{Agradecimentos}

Os autores agradecem ao CNPq (Conselho Nacional de Desenvolvimento Científico e Tecnológico) pela bolsa concedida à primeira autora (P. X. B.) e à FAPEMIG (Fundação de Amparo à Pesquisa do Estado de Minas Gerais) pelo suporte financeiro ao projeto.

\section{Referências}

Aldrigue, M. L. (1998). Desenvolvimento e validação de metodologia analítica, utilizando a CLAE, para determinação de vitamina $C$ em frutas e seus principais produtos. Tese de Doutorado, Universidade Estadual de Campinas, Campinas, SP, Brasil.

Al-Ghannam, S. M.; Al-Olyan, A. M. (2005). Differential Electrolytic Potentiometric Titration of Vitamin C in Pharmaceutical Preparations. Journal of Food and Drug Analysis, 13(4), 295-300. https://doi,org/10.38212/2224-6614.2574

Aydogmus, Z.; Cetin, S. M.; Özgür, M. U. (2002). Determination of Ascorbic Acid in Vegetables by Derivative Spectrophotometry. Turkish Journal of Chemistry, 26(5), 697-704. https://dergipark.org.tr/tr/pub/tbtkchem/issue/11944/142862

Bueno, D. M. A.; Gomes, S. I. A. A.; Giust, E. D.; Stadler, J. P. (2019). Determinação da vitamina $\mathrm{C}$ em suco de laranja: uma proposta experimental investigativa para aplicação no ensino de química. Revista Brasileira de Ensino de Ciências e Tecnologia, 12(3), 307-325.

https://doi.org/10.3895/rbect.v12n3.8674

Emadi-Konjin, P.; Verjee, Z.; Levi, A. V.; Adeli, K. (2005). Measurement of intracellular vitamin $\mathrm{C}$ levels in human lymphocytes by reverse phase high performance liquid chromatography (HPLC). Clinical Biochemistry, 38(5), 450-456. https://doi.org/10.1016/j.clinbiochem.2005.01.018 Faria, R. B. (1995). Introdução aos Sistemas Químicos Oscilantes, Química Nova, 18(3), 281-294. http://static.sites.sbq.org.br/quimicanova.sbq.org.br/pdf/Vol18No3_281_v18_n3_08.pdf

Maia, L. F. O.; Reis, E. L.; Reis, C.; Reis, C. D. G.; Damascenos, O. I. C.; Reis, L. G. T. (2019). Potentiometric Determination of Paracetamol in Pharmaceutical Formulations by the Analyte Pulse 
Perturbation Technique Using Belousov-Zhabotinskii Oscillating Chemical Reaction. Journal of Analytical Chemistry, 74(12), 1232-1238. https://doi.org/10.1134/S1061934819120074

Ojani, R.; Raoof, J.; Mahdavi, F. (2003). The potentiometric effect of As(III) ion on a BelousovZhabotinskii oscillating chemical reaction. Application to the determination of As(III), Bulletin of the Chemical Society of Japan, 76(11), 2117-2121. https://doi.org/10.1246/bcsj.76.2117

Oliveira, R. G.; Godoy, H. T.; Prado, M. A. (2010). Optimization of a colorimetric method to determine ascorbic acids in fruit jelly. Food Sci. Technol, 30(1), 244-249. https://doi.org/10.1590/S0101$\underline{20612010000100036}$

Paim, A. P. S.; Almeida, C. M. N. V.; Reis, B. F.; Lapa, R. A. S.; Zagatto, E. A.G.; Lima, J. L. F. C. (2002). Automatic potentiometric flow titration procedure for ascorbic acid determination in pharmaceutical formulations, Journal of Pharmaceutical and Biomedical Analysis, 28(6), 12211225. https://doi.org/10.1016/s0731-7085(02)00018-3

Raoof, J.; Ojani, R.; Kiani, A. (2004). Kinetic Determination of Silver Ion by Its Perturbation on Belousov-Zhabotinskii Oscillating Chemical Reaction Using Potentiometric Method, Analytical Sciences, 20(5), 883-886. https://doi.org/10.2116/analsci.20.883

Yebra-Biurrun, M. C. (2000). Flow injection determination methods of ascorbic acid, Talanta, 52(3), 367-383. https://doi.org/10.1016/s0039-9140(00)00402-1 